\title{
Implementation of an enhanced discharge teaching protocol to reduce 30-day hospital readmissions in adults diagnosed with Sickle Cell Disease
}

\author{
Jean Thorpe-Williams ${ }^{1}$, Diana-lyn Baptiste*1, Joanne E. Jarboe-Costello ${ }^{2}$, Sharon Kozachik ${ }^{3}$ \\ ${ }^{1}$ School of Nursing, The Johns Hopkins University, Baltimore, Maryland, USA \\ ${ }^{2}$ Nursing Education at the Holy Cross Hospital in Silver Spring, Maryland, USA \\ ${ }^{3}$ College of Nursing, Medical University of South Carolina, USA
}

Received: November 30, 2020

DOI: $10.5430 /$ jnep.v11n6p60
Accepted: February 7, 2021

Online Published: February 28, 2021

\begin{abstract}
Background and objectives: Sickle Cell Disease is a painful genetic disorder characterized by abnormal hemoglobin that affects 1-in-365 African American births. Individuals with Sickle Cell Disease often experience frequent hospitalizations, resulting in 30-day readmission rates 2.5 times higher than those without Sickle Cell Disease. Discharge education programs have shown to improve 30-day readmissions and improve health outcomes.

Methods: This advanced practice nurse-led initiative incorporated components of the Re-Engineered Discharge (Project RED) protocol, which included patient and medication education, specific discharge teaching, and post-discharge follow-up phone calls over a 12-week period. A pre/posttest design was used to evaluate 30-day readmission. Analyses included descriptive statistics and Fisher's exact test.

Results: Of the $(\mathrm{N}=10)$ participants, eight $(80 \%)$ were female, with a mean age of $31.08( \pm 4.33)$ and all were African Americans. Although not statistically significant, there was a $48 \%$ reduction in 30 -day readmissions rates between the pre/post intervention periods.

Conclusions: The utilization of an enhanced discharge teaching protocol is a practical solution to increase patients' readiness for discharge and to reduce 30-day readmission rates. Project RED discharge teaching protocol is an effective strategy for nurses to employ to improve patient outcomes and quality of care for persons with Sickle Cell Disease.
\end{abstract}

Key Words: Sickle Cell Disease, Readmission, Vaso-occlusive crisis, Patient education, discharge teaching, Hospitalization

\section{INTRODUCTION}

Sickle cell disease (SCD) is an inherited genetic disorder that occurs in one out of every 365 Black or African-descent American births and affects approximately 100,000 Americans in the United States. ${ }^{[1,2]}$ SCD is diagnosed based on the appearance of abnormal hemoglobin $\mathrm{S}$, which causes red blood cells to become stiff and abnormally shaped. This re- sults in reduced oxygen-carrying capacity, slowed or stopped blood flow, all of which can lead to vaso-occlusive crises. ${ }^{[1]}$ Vaso-occlusive crises are the most prevalent manifestations of SCD and are precipitated by conditions such as dehydration, illness, stress, high altitudes, or weather temperature changes. ${ }^{[2]}$ The management and treatment of vaso-occlusive crises include frequent hospitalizations, which can result in USA. 
high hospital readmission rates.

Thirty-day readmissions are defined by the Centers for Medicare and Medicaid Services as a return to hospitalization in an acute care setting following a prior acute care admission within 30 days. $^{[3,4]}$ Consequently, Centers for Medicare and Medicaid Services has established 30-day readmission rates as an indicator of quality care and has established the Hospital Readmission Reduction Program, which reduces payments to hospitals with excessive readmission rates. ${ }^{[3,4]}$ In prior years, frequent readmissions were initially thought to be related to the inevitable vaso-occlusive crises associated with SCD. However, frequent readmissions are now better understood and are often related to suboptimal level of care of persons with SCD. ${ }^{[5,6]}$ Along with the negative health outcomes for patients with SCD, statistics for frequent readmissions show that there is also an increased financial burden for healthcare institutions. The estimated cost of care for the 100,000 individuals with SCD is more than $\$ 1$ billion annually in the United States. ${ }^{[4,5,7,8]}$

Healthcare institutions continuously seek best practices to reduce high readmission rates and improve care for the patients they serve. This is in alignment with the principal aims of Centers for Medicare and Medicaid Services, which include better care, healthier people, and smarter spending. ${ }^{[4]}$ Additionally, the Agency for Healthcare Research and Quality recommends that improving the quality and safety of hospitals' discharge processes and care transitions can lead to reductions in potentially avoidable readmissions. ${ }^{[9]} \mathrm{Re}-$ search findings have shown that improving the discharge process and making it more individualized and specific to the patients' needs can reduce readmissions. ${ }^{[5,7,10]}$ Therefore, incorporating enhanced discharge teaching in the care of persons with SCD may improve the quality of care and reduce 30-day readmission rates.

Persons with SCD who experience frequent hospital readmissions due to vaso-occlusive crises typically have a poor quality of life, decreased career productivity, and poor overall health outcomes. ${ }^{[7]}$ The quality of life for persons with sickle cell disease is adversely affected by depression, difficulties with paying bills, and lower socioeconomic status. ${ }^{[11]}$ Some clinicians equate frequent readmissions with malingering or drug-seeking behaviors. ${ }^{[5,11]}$ These biases may lead to premature discharges and inadequate follow-up, which further contribute to the cycle for readmissions. ${ }^{[5]}$ Being discharged from the hospital often does not indicate the end of the crisis for persons with SCD. ${ }^{[5]}$

Persons diagnosed with SCD have 30-day readmission rates that are higher than those seen among persons diagnosed with

Published by Sciedu Press asthma or diabetes. ${ }^{[2]}$ In 2016, SCD-related 30-day readmission rates were an alarming $31.1 \%$, compared with $12.5 \%$ for non-SCD related readmissions. ${ }^{[12]}$ Adults aged 18-30 years old had the highest incidence of readmissions. ${ }^{[4,7,13]}$ In 2017, the 30-day readmission rates for persons admitted with vaso-occlusive crises due to SCD at a Mid-Atlantic region metropolitan community hospital within a health care system was $38.24 \%$, compared to the national rate of $31.1 \%$. A review of this hospital's discharge teaching process identified the need for improvement. There was no specific discharge process that addressed the individualized needs of this patient population. Improving the discharge process by implementing specific interventions is a feasible strategy for reducing readmissions. ${ }^{[9-11,14,15]}$

Optimizing the use of evidence-based practice to improve the discharge process can reduce 30-day readmission rates and reduce the negative consequences associated with them. Evidence-based practice is key to achieving the quadruple aim, which is a framework that serves as a foundation to optimize health system performance. ${ }^{[16]}$ The quadruple aim seeks to improve the admitted person's experience, improve population health, reduce cost of care, and improve the worklife of healthcare providers. ${ }^{[16,17]}$ Reducing 30-day readmissions rates for young adults with SCD can have a meaningful impact on both admitted persons and the institution. These endeavors further contribute to achieving the goals of the quadruple aim.

The purpose of this quality improvement project was to evaluate the effect of a nurse-led evidence-based discharge teaching education program on 30-day readmissions in adults admitted for SCD. The objectives of this project were to implement a nurse-led discharge education program and evaluate 30-day readmission rates among patients admitted with SCD.

\section{Methods}

\subsection{Design, setting, and sample}

For this quality improvement project, an advanced practice nurse led the initiative to incorporate an evidence-based discharge process. The pre-post project sought to improve the discharge teaching process and reduce 30-day readmission rates for patients admitted with SCD. The project was implemented over a 12-week time period, from October 2019 to December 2019 at a 449-bed metropolitan community hospital located in the Mid-Atlantic region of the United States. Data collection was conducted on a 30-bed hematology/oncology unit with a population consisting predominantly of adults admitted with SCD or cancer diagnoses. 


\subsection{Theoretical framework}

The translation framework that guided this quality improvement project was the Knowledge-to-Action Model (see Figure 1). The Knowledge-to-Action framework allowed us to focus on the relationships among the problem of high 30-day readmission rates in persons with $\mathrm{SCD}$, the processes, the outcomes and sustaining the knowledge for long-term use. In the Knowledge-to-Action framework, knowledge is conceptualized as moving through a funnel until it is translated and used ${ }^{[18]}$ Knowledge-to-Action framework is composed of two distinct, but related components: Knowledge Creation and Action Cycle. ${ }^{[18]}$ These two components ensured that there was a systematic process with feedback loops that we were able to utilize in implementing an enhanced discharge teaching protocol to reduce 30-day readmissions rates in persons with SCD.

In the knowledge creation phase, we identified gaps in the current discharge teaching for persons admitted with SCD and reviewed the literature, which supported the use of enhanced discharge teaching protocols as means of reducing 30-day readmission rates. The Re-Engineered Discharge (Project RED) protocol, an evidence-based intervention, has been shown to reduce 30-day readmission rates. ${ }^{[9,14,15,19]}$ After adapting the knowledge to our problem of high 30-day readmission rates in our patient population, it was important to assess the barriers. Some of the barriers identified as threats to the success of the project were: time constraints for nurses due to competing priorities of also caring for oncology patients, resistance to change, limited knowledge of using evidenced-based guidelines to manage persons with SCD, and high nurse/patient ratio.

The action cycle phase is comprised of several phases that are fundamental in translating knowledge into practice. The phases include identification of the problem, seeking out knowledge relevant to solving the problem, and adapting it to the local context. The next step involved assessing potential barriers that may limit utilizing the knowledge and then incorporating strategies to mitigate barriers and promote knowledge utilization. ${ }^{[18]}$

The final steps included implementing the interventions, monitoring the outcomes and sustaining knowledge utilization. During project implementation, we incorporated components of Project RED to enhance the current discharge teaching for persons admitted with SCD. Specific interventions were implemented, such as teaching patients about their disease and medications, making outpatient follow-up appointments before discharge and 48 hours post-discharge follow-up phone calls. This quality improvement project implemented components of Project RED over 12 weeks. It then measured nurses' adherence to using Project RED and 30-day readmission rates pre- and post-intervention.

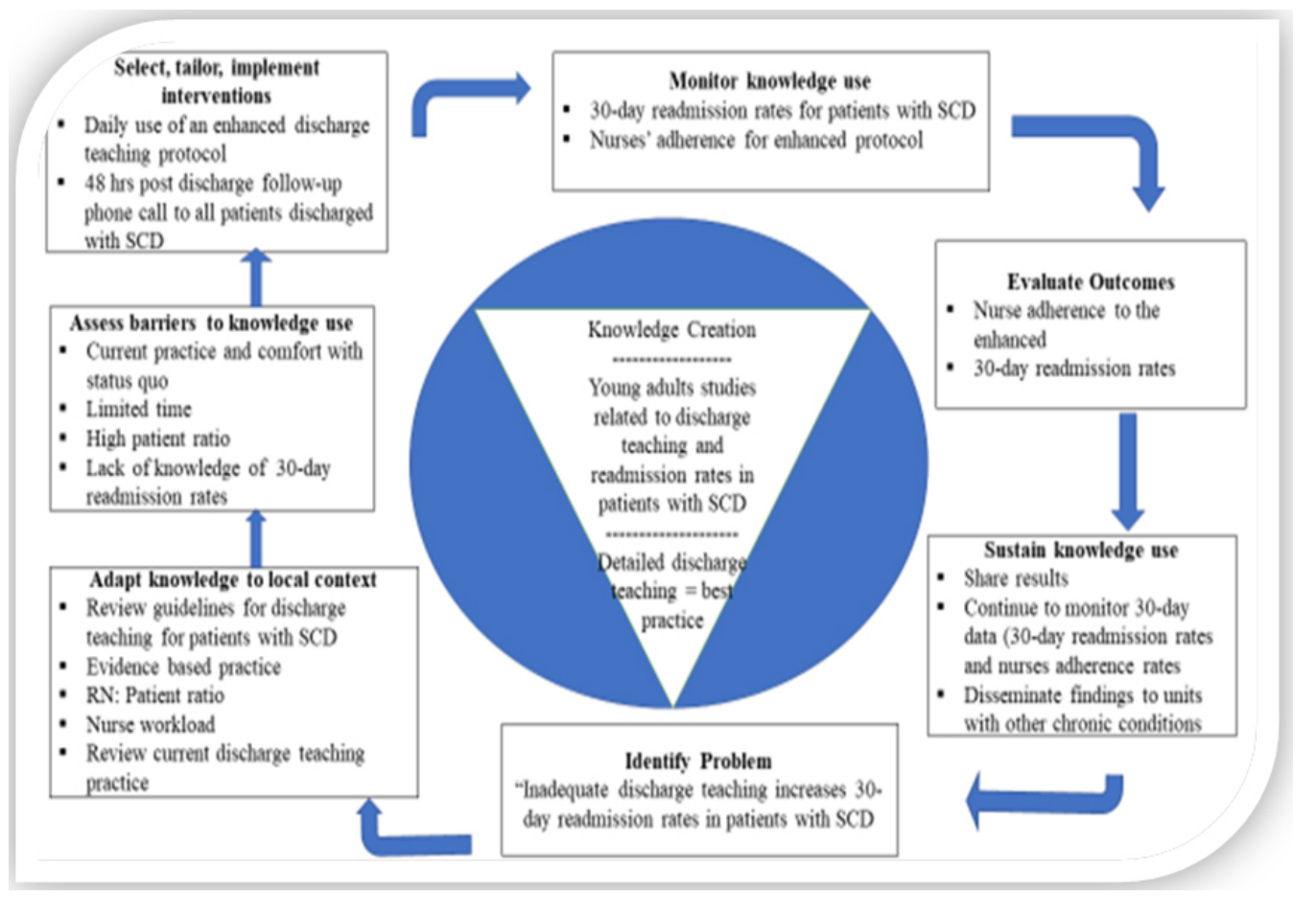

Figure 1. Knowledge-to-action model

Adapted from The Knowledge to Action Framework. From Graham I, Logan J, Harrison M, Strauss S, Tetroe J, Caswell W, Robinson N: Lost in knowledge translation: time for a map? The Journal of Continuing Education in the Health Professions. 2006; 26: 13-24. 


\subsection{Intervention: Project RED protocol}

The Project RED protocol is a nationally recognized evidence-based initiative shown to reduce 30-day readmission rates. ${ }^{[9,10,15]}$ Project RED has components that enhance the discharge process such as comprehensive discharge teaching, disease and medication management, patient education, and post-discharge follow-up. ${ }^{[9,10,15]}$ One of the guiding principles of Project RED is that all patients should leave the hospital with an easy-to-understand discharge plan. This is accomplished with the After-Hospital Care Plan, which encourages hospitalized persons to become more active participants in their care. In addition, the After-Hospital Care Plan ensures that patients are better able to take care of themselves when they go home. ${ }^{[9]}$ Project RED also uses discharge educators to provide post-discharge follow-up telephone calls to assess clinical status and to reinforce the plan of care. Project RED has been successful in improving patients' communication and understanding of clinical outcomes, enhanced discharge preparation, and scheduling follow-up appointments. ${ }^{[9,10,15]}$ The success of Project RED led to a partnership between Boston University Medical Center, who are the developers, and the Agency for Healthcare Research and Quality to disseminate the program nationwide. ${ }^{[20]}$

\subsection{Nurse-led implementation}

For this quality improvement project, nurses performed more thorough and detailed discharge teaching with the use of the components of Project RED. The components included the following: (1) educating person with SCD on management and strategies to prevent and reduce vaso-occlusive crises, (2) smoking cessation, (3) medication management, and (4) providing copies of educational material on SCD. The AfterHospital Care Plan and the Discharge Educator components of Project RED was incorporated into the unit-based discharge teaching to 10 patients over a 12 -week time period. Nurses received training on the Project RED intervention. The After-Hospital Care Plan handout provided interventions specific to SCD, focused on medication management, dietary and exercise recommendations, smoking cessation recommendations and encouraging early post-discharge follow-up appointments $^{[19]}$ (see Figure 2). A trained discharge nurse educator involved with the project made follow-up telephone call within 48 hours of each person being discharged. During the follow-up telephone call, ten questions to reinforce discharge instructions. The post-discharge follow up checklist was adapted from the After Hospital Care Plan section of the Project RED protocol, which included inquiries regarding the receipt of SCD education, follow-up appointments, and medication reconciliation (see Figure 3). Nurses were provided with daily reinforcement and support using the enhanced discharge teaching protocol. All the trained nurses were expected to utilize the discharge teaching with all patients who received the education program.

\subsection{Ethical considerations}

This study was reviewed by the organization's Institutional Review Board and was deemed as quality improvement.

Informed consent was not required because the intervention was integrated into usual care for all patients admitted for vaso-occlusive crises related to SCD.

\subsection{Measures}

The outcome measure 30-day readmission rates. The effectiveness of the intervention was evaluated by comparing the 30-day readmission rates before and after implementing the enhanced discharge-teaching program.

\subsection{Analysis}

Descriptive statistics were used to summarize sociodemographic characteristics for individuals admitted with SCD. A Fisher's Exact Test was used to compare 30-day readmission rates before and after the intervention. Statistical analysis was performed using SPSS ${ }^{\circledR}$ software version 24. ${ }^{[21]}$

\section{Results}

\subsection{Socio demographic characteristics}

Of the $(\mathrm{N}=10)$ participants, eight $(80 \%)$ were female, with a mean age of $31.08( \pm 4.33)$ and all were African Americans. The highest level of education completed among the sample was "some college", while only two of the ten participants reported having full-time employment (see Table 1).

\subsection{Thirty-day readmission rates}

During the 12-week implementation period, there were two of 10 patients $(20 \%)$ who received the Project RED education and, were readmitted within 30-days. Eight of the ten participants responded to the follow-up phone calls. Of the eight participants who responded to follow-up calls, one was readmitted within 30-day. The other participant who was readmitted within 30-days was between the two participants who did not receive the follow-up call. In the 2017, comparison groups using treatment as usual showed 65 of 170 patients (38.24) were readmitted. A percentage change calculator was used to compare readmission rates between the pre and post intervention periods. This represented a $48 \%$ reduction in readmission rates for patients receiving the discharge teaching protocol. Although there was a decrease in readmissions between both periods, a Fishers Exact test showed there was no significant difference in the proportion of persons readmitted $(p=.329)$. 


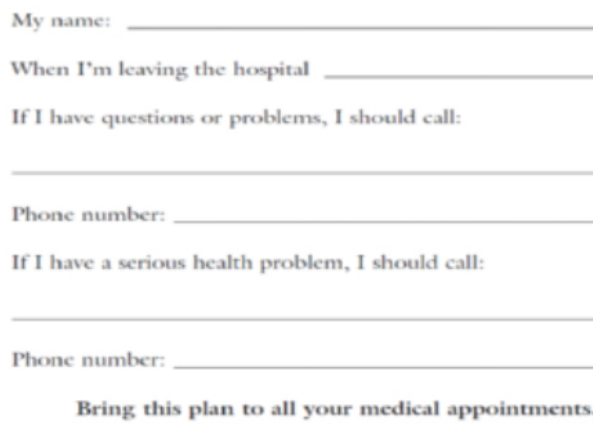

What activitics or foods should I avoid?
What medicines do I need to take?

Each day, follow this schedule:

\begin{tabular}{|c|c|c|c|}
\hline \multicolumn{4}{|c|}{ Morning Medicines } \\
\hline 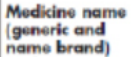 & 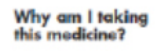 & 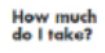 & $\begin{array}{l}\text { How dol alake } \\
\text { this modicher }\end{array}$ \\
\hline & & & \\
\hline & & & \\
\hline & & & \\
\hline & & & \\
\hline & & & \\
\hline & & & \\
\hline & & & \\
\hline & & & \\
\hline & & & \\
\hline
\end{tabular}

Reference: AHRQ, Project RED After Hospital Care Plan https://www.ahrq.gov/sites/default/files/wysiwvg/patients-consumers/diagnosistreatment/hospitals-clinics/goinghome/goinghomeguide.pdf

Figure 2. Managers' and nurses' competence assessments

\begin{tabular}{|c|c|c|c|c|}
\hline Attempt \#1 & Date \& Time & Reached ___ Yes ___ No & \multirow[b]{3}{*}{ Yes } & \multirow[b]{3}{*}{ No } \\
\hline \multirow[t]{2}{*}{ Attempt \#2 } & \multirow[t]{2}{*}{ Date \& Time } & Reached ___ Yes ___ No & & \\
\hline & & & & \\
\hline \multicolumn{3}{|c|}{ 1. Patient received discharge plan with information and reasons for admission. } & & \\
\hline \multicolumn{3}{|c|}{ 2. Patient received written information on discharge related to vaso-occlusive crisis SCD } & & \\
\hline \multicolumn{3}{|c|}{ 3. Patient received information on early identification of triggers to reduce vaso-occlusive crises in SCD } & & \\
\hline \multicolumn{3}{|c|}{ 4. Patient knew when to call the doctor } & & \\
\hline \multicolumn{3}{|c|}{ 5. Patient had follow-up appointment scheduled for sickle cell disease management prior to discharge. } & & \\
\hline \multicolumn{3}{|c|}{ 6. Patient received written information on medication (s) and understood the purpose of his/her medications. } & & \\
\hline \multicolumn{3}{|c|}{ 7. Patient knew when to take medications } & & \\
\hline \multicolumn{3}{|c|}{ 8. Patient told about test results } & & \\
\hline \multicolumn{3}{|c|}{ 9. Patient at high risk of readmission has follow-up phone calls scheduled prior to discharge. } & & \\
\hline \multicolumn{3}{|c|}{ 10. Was the patient admitted in the past 30 days? } & & \\
\hline
\end{tabular}

Adapted from Project RED ${ }^{[9]}$

Figure 3. Managers' and nurses' competence assessments 
Table 1. Sociodemographic characteristics of participants admitted with SCD

\begin{tabular}{ll}
\hline Items & N (\%) \\
\hline Age, mean (SD) & $31.08( \pm 4.33)$ \\
Gender & $2(20)$ \\
Male & $8(80)$ \\
Female & \\
Highest Level of Education Completed & $4(40.0)$ \\
High School & $2(20.0$ \\
Some College & $4(40.0)$ \\
No response & \\
Employment Status & $6(60.0)$ \\
Unemployed & $2(20.0)$ \\
Employed & $2(20.0)$ \\
No response & \\
Race & $10(100)$ \\
African American & \\
\hline Note. SD = standard deviation
\end{tabular}

\section{Discussion}

The reduction of hospital readmission rates may be improved by the implementation of an evidence-based enhanced discharge-teaching program. The purpose of this quality improvement project was to evaluate to use of Project RED discharge education on 30-day readmission rates. Although the results were not statistically significant, findings in this project suggest that readmissions can be reduced by implementing an enhanced discharge teaching and support process. These results are similar to findings from previous studies that showed Project RED was successful in reducing 30-day readmission rates. ${ }^{[14,15,22,23]}$ Although participants' knowledge and satisfaction were not measured, their responses during the follow-up telephone calls reflected improved understanding, and increased satisfaction with the enhanced discharge process.

Through this project, the authors identified barriers and gaps in the discharge process. Most notable was the lack of discharge education and adequate follow-up care when persons with SCD were discharged from the hospital. Consistent with previous studies, Project RED interventions was used to improve outcomes by targeting key recommendations for the discharge process: (1) encouraging persons to become more involved in their discharge process, (2) ensuring primary providers received discharge transition records sooner, (3) increasing referrals for home health care services, and (4) increasing follow-up with primary providers. ${ }^{[10,14,15,22]}$ Identifying of these barriers and gaps provided opportunities to improve the discharge process and overall care, and ultimately reduce readmissions.

Published by Sciedu Press
In previous studies, Project RED has been successful in reducing 30-day readmission rates by enhancing the discharge process not only for all-cause readmission but also for the three penalized Centers for Medicare and Medicaid conditions: congestive heart failure, acute myocardial infarction, and pneumonia. ${ }^{[9,10,14,15]}$ Additionally, Project RED has led to the following: endorsed readiness for discharge; reduced costs by $\$ 412$ per person; branded the hospital as a highquality facility; and improved persons and family satisfaction. ${ }^{[15]}$ These results indicate that improving the discharge process can ultimately increase patients' health outcomes, reduce all-cause mortality, and reduce the financial burden for health care.

The role of the nurses in the collaboration with patients and caregivers have been found to have a positive correlation on patients and caregivers' knowledge of their disease process, medications and compliance treatment plan and follow-up care. ${ }^{[10]}$ Nurses were able to incorporate the enhanced discharge teaching of Project RED in usual care. Similar to other studies, our study demonstrated that with the use of Project RED protocol, nurse were instrumental in reducing hospital utilization, improving patient preparation for discharge and increasing follow-up care after discharge.

\section{Limitations}

The implementation of an enhanced discharge teaching protocol on a busy hematology unit presented some challenges and limitations. One major limitation was the small sample size of participants. This was primarily due to the inclusion criteria of using only participants admitted to one unit. Additionally, the small sample size was due to low-census in SCDrelated hospital admissions over the 12 -week period. Patients with SCD are more likely to experience vaso-occlusive crises [requiring hospitalization] in the colder, winter months. ${ }^{[2]}$ Future research is suggested to formally test the efficacy of an enhanced discharge teaching and support intervention. Despite these limitations, this quality improvement project demonstrated that nurses were instrumental in improving the discharge process and overall care of persons with SCD and that patients had fewer readmissions with the enhanced discharge teaching.

\section{Conclusion}

High readmission rates continue to be a significant challenge in the successful management of patients with chronic conditions, such as SCD. Improving the discharge process is an effective strategy that can reduce readmission rates and improve the overall care of persons with SCD. This quality improvement project implemented an enhanced discharge teaching protocol to reduce high 30-day readmission rates 
in persons with SCD. Components of Project RED such as disease and medication education, scheduling post-discharge appointments and post-discharge follow-up telephone calls were implemented to enhance the discharge process. These components are all nurse-driven interventions that have been shown to improve the discharge process. Tailoring the dis- charge process to the specific needs of persons with SCD can help better prepare them for discharge, improve health outcomes, and ultimately reduce hospitalizations and overall healthcare costs.

\section{CONFLicts OF InTEREST Disclosure}

The authors declare that there is no conflict of interest.

\section{REFERENCES}

[1] Yawn BP, Buchanan GR, Afenyi-Annan AN, et al. Management of sickle cell disease: summary of the 2014 evidence-based report by expert panel members. Jama. 2014; 312(10): 1033-48. PMid:25203083 https://doi.org/10.1001/jama.2014.10517

[2] Institute NHLaB. Evidence-based management of sickle cell disease: expert panel report, 2014. 2020 [cited 2020 November 1]. Available from: https://www.nhlbi.nih.gov/health-topics/eviden ce-based-management-sickle-cell-disease

[3] Catalyst N. Hospital Readmissions Reduction Program (HRRP). NEJM Catalyst. 2018.

[4] Kumar V, Chaudhary N, Achebe MM. Epidemiology and Predictors of all-cause 30-Day readmission in patients with sickle cell crisis. Sci Rep. 2020; 10(1): 2082. PMid:32034210 https ://doi.org/10.1 038/s41598-020-58934-3

[5] Naymagon L. Reducing Readmission in Sickle Cell Disease: The Role of the Patient-Centered Medical Home.

[6] Sop Mouaffo D, Smith WR, Owens Z, et al. Reducing Hospital Use of Urban Sickle Cell Adults Using Multidisciplinary Adult Care and Instrumental Support. Blood. 2017; 130(Supplement 1): 5661-.

[7] Brodsky MA, Rodeghier M, Sanger M, et al. Risk Factors for 30-Day Readmission in Adults with Sickle Cell Disease. Am J Med. 2017 130(5): 601.e9-.e15. PMid:28065771 https://doi.org/10.101 $6 / j$.amjmed.2016.12.010

[8] Lin RJ, Evans AT, Wakeman K, et al. A Mixed-Methods Study of Pain-related Quality of Life in Sickle Cell VasoOcclusive Crises. Hemoglobin. 2015; 39(5): 305-9. PMid:26114739 https://doi.org/10.3109/03630269.2015.1055576

[9] Quality AfHRa. Re-Engineered Discharge (RED) Toolkit. 2020 [cited 2020]. Available from: https://www.ahrq.gov/patient-saf ety/settings/hospital/red/toolkit/index.html

[10] Adams CJ, Stephens K, Whiteman K, et al. Implementation of the Re-Engineered Discharge (RED) toolkit to decrease all-cause readmission rates at a rural community hospital. Qual Manag Health Care. 2014; 23(3): 169-77. PMid:24978166 https ://doi .org/10.109 7/QMH. 0000000000000032

[11] Cronin RM, Hankins JS, Byrd J, et al. Risk factors for hospitalizations and readmissions among individuals with sickle cell disease: results of a U.S. survey study. Hematology. 2019; 24(1): 18998. PMid:30479187 https ://doi.org/10.1080/16078454. 201 8.1549801

[12] Fingar KR, Owens PL, Reid LD, et al. Characteristics of Inpatient Hospital Stays Involving Sickle Cell Disease, 2000-2016: Statistical Brief \#251. Healthcare Cost and Utilization Project (HCUP) Statis- tical Briefs. Rockville (MD): Agency for Healthcare Research and Quality (US); 2006.

[13] Nouraie M, Gordeuk VR. Blood transfusion and 30-day readmission rate in adult patients hospitalized with sickle cell disease crisis. Transfusion. 2015; 55(10): 2331-8. PMid:26126756 https : //doi.org/10.1111/trf.13155

[14] Mitchell SE, Martin J, Holmes S, et al. How Hospitals Reengineer Their Discharge Processes to Reduce Readmissions. J Healthc Qual. 2016; 38(2): 116-26. PMid:26042743 https ://doi.org/10.109 7/JHQ.0000000000000005

[15] Jack BW, Chetty VK, Anthony D, et al. A reengineered hospital discharge program to decrease rehospitalization: a randomized trial Ann Intern Med. 2009; 150(3): 178-87. PMid:19189907 https : //doi.org/10.7326/0003-4819-150-3-200902030-00007

[16] Lavenberg JG, Cacchione PZ, Jayakumar KL, et al. Impact of a Hospital Evidence-Based Practice Center (EPC) on Nursing Policy and Practice. Worldviews Evid Based Nurs. 2019; 16(1): 4-11. PMid:30714308 https ://doi.org/10.1111/wvn. 12346

[17] Bodenheimer T, Sinsky C. From triple to quadruple aim: care of the patient requires care of the provider. Ann Fam Med. 2014; 12(6): 573-6. PMid:25384822 https ://doi.org/10.1370/afm.1713

[18] Graham ID, Logan J, Harrison MB, et al. Lost in knowledge translation: time for a map? J Contin Educ Health Prof. 2006; 26(1): 13-24. PMid:16557505 https ://doi.org/10.1002/chp. 47

[19] Patel PH, Dickerson KW. Impact of the Implementation of Project Re-Engineered Discharge for Heart Failure patients at a Veterans Affairs Hospital at the Central Arkansas Veterans Healthcare System. Hosp Pharm. 2018; 53(4): 266-71. PMid:30038447 https : //doi.org/10.1177/0018578717749925

[20] Shan D, Finder J, Dichoso D, et al. Interventions to prevent heart failure readmissions: The rationale for nurse-led heart failure programs. Journal of Nursing Education and Practice. 2014; 4(11): 23 32. https://doi.org/10.5430/jnep.v4n11p23

[21] Corporation I. IBM SPSS Statistics for Windows, Version 26.0. Armonk, NY; 2019.

[22] Sullivan JL, Shin MH, Engle RL, et al. Evaluating the Implementation of Project Re-Engineered Discharge (RED) in Five Veterans Health Administration (VHA) Hospitals. Jt Comm J Qual Patient Saf. 2018; 44(11): 663-73. PMid:30097383 https://doi.org/10.1 016/j.jcjq. 2018.01.007

[23] Shekelle PG, Pronovost PJ, Wachter RM, et al. Advancing the science of patient safety. Ann Intern Med. 2011; 154(10): 6936. PMid:21576538 https://doi.org/10.7326/0003-4819-1 54-10-201105170-00011 